\title{
Three Potential Problems for Powers' One-Fallacy Theory
}

\author{
Matrhew Zuckero Wayne State University
}

\begin{abstract}
Lawrence Powers advocates a one-fallacy theory in which the only real fallacies are fallacies of ambiguity. $\mathrm{He}$ defines a fallacy, in general, as a bad argument that appears good. He claims that the only legitimate way that an argument can appear valid, while being invalid, is when the invalid inference is covered by an ambiguity. Several different kinds of counterexamples have been offered from begging the question, to various forms of ad hominem fallacies. In this paper, I outline three potential counterexamples to Powers' theory, including one that has been addressed already by Powers, and two which are well known problems, but until now have never been applied as counterexamples to Powers' theory. I argue that there is a simpler explanation of these 'hard' cases than positing ambiguities that are not obviously there.
\end{abstract}

Résumé: Lawrence Powers préconise une théorie d'un sohphisme-unique dans laquelle les sophismes d'ambiguïté sont les seuls véritables sophismes. Selon sa définition générale, un sophisme est un mauvais argument qui semble bon. Il affirme que c'est seulement à cause d'une ambiguïté qu'un argument fallacieux déductif ou inductif peut paraître convaincant. On a déjà avancé contre sa théorie des contre-exemples tels que la pétition de principe et diverses formes du sophisme ad hominem. Dans cet article je trace les grandes lignes de trois contre-exemples potentiels, y compris un contre-exemple que Powers a déjà addressé, mais les deux autres sont des problèmes bien connus qui jusqu'à présent n'ont jamai été employés comme contre-exemples contre sa théorie. Je soutiens qu'il y a une explication plus simple que d'avancer des ambiguïtés qui n'existent même pas.

Keywords: Powers, equivocation, one-fallacy theory, dividing by zero, Wason, Monty Hall

\section{Introduction}

Lawrence Powers advocates a one-fallacy theory in which the only real fallacies are fallacies of ambiguity. He defines a fallacy, in general, as a bad argument that appears good. He claims that the only legitimate way that a deductive argument can appear valid, while being invalid, is when the invalid inference is covered by an ambiguity. The analogous framework will, of course, apply to inductive arguments as well. Weak inductive fallacies will appear strong because of some ambiguity in the argument. The reason that Powers' focuses on deductive arguments in most of his present published work, is because he sees philosophy as a broadly deductive enterprise in his metaphilosophy.

So what kinds of commonly named fallacies are not in fact fallacies on Powers' One-Fallacy Theory? So-called fallacies of 'affirming the consequent' and 'denying the antecedent' are "nakedly" invalid and thus do not appear valid at all. His project seems to be at least partially motivated by the desire to have a unified notion of 
what a fallacy is. According to him the notion of fallacy, as it presently stands, is loose and imprecise; almost any kind of dialectical, methodological or logical mistake counts as a fallacy. In a series of papers he defends this thesis. Several different kinds of counterexamples have been offered from begging the question to various forms of ad hominem fallacies. In this paper, I outline three potential counterexamples to Powers' theory and I argue that there is a simpler explanation of these 'hard' cases than positing ambiguities that are not obviously there. First, I will give the cases; second, I will outline my alternate explanation of the fallaciousness of these cases and argue that it is simpler and more plausible, and finally I will give an argument showing why these are, in fact, counterexamples to Powers' theory.

Before I begin this discussion I want to lay out Powers' rationality- $1 /$ rationality2 distinction. Rationality-1 is an idealization of what it is to be rational. A person that possess rationality-1 is a person with an infallible grasp of all concepts, who would never commit a fallacy or intellectual mistake of any kind. We are obviously not rational-1, for we do indeed make mistakes in reasoning and lack complete grasps of many concepts. We possess rationality-2. We have a fallible grasp of most concepts. We don't clearly understand them, and this leads us to make many mistakes when we attempt to implement them. Though we can never attain rationality-1, we have some idea of what rationality-1 would be like, for we have the ability to recognize when we make mistakes. In striving to attain rationality-1, we learn some important rules along the way. This allows us to keep things that we normally call irrational from being rational. In other words, not just anything counts as rational -2 ; there are some standards that have to be met. For example, it would not be rational-2 to hold inconsistent beliefs or believe in contradictions. To sum up, rationality- 1 represents infallible perfect understanding of concepts, whereas rationality-2 represents fallible, less than perfect, understanding of concepts.

In "Dividing by zero and other mathematical fallacies," Powers discusses an apparent counterexample to his theory that stems from a certain case involving division by zero. For example, consider the following argument,
1) Let $a=b$
2) $\quad a^{2}=a b$
(multiply both sides by a)
3) $a^{2}-b^{2}=a b-b^{2} \quad$ (subtract $b^{2}$ from each side)
4) $\quad(a+b)(a-b)=b(a-b)$ (factor both sides)
5) $\quad(a+b)=b$
6) $2 b=b$
(cancel $(a-b)$ on both sides)
7) $2=1$
(since $a=b$ )
(canceling $b$ on both sides)

Now, the invalid step occurs between 4 and 5 , because $a-b=0$, and one cannot divide by zero. Powers admits that this is a fallacy, for it passes his phenomenological test. It definitely appears valid in legitimate way. On Powers' view, it seems that an argument appears valid in a legitimate way if and only if it 
appears to comport with the rules of logic. In other words, just because ordinary people (untrained in the skills of reasoning) are 'pulled in' by a mistake does not mean that a fallacy has been committed, even if the argument is invalid. Powers also admits that in the above argument there is, prima facie, no apparent ambiguity covering the invalid step from 4 to 5 ; it seems to be a naked mistake and thus is an apparent counterexample to his theory. In contrast, in the following argument there does appear to be, prima facie, an ambiguity at work:

(1) Rivers run.

(2) Anything that runs has feet.

(3) Therefore, rivers have feet.

To account for this Powers argues that there is an ambiguity in the notion of 'number', and that the inference relies on this ambiguity and subsequently, it is a fallacy of ambiguity that makes the invalid step appear as though it is valid. His theory survives unscathed. He says:

And so one fallacious way of dividing by zero would be to apply the true law that division is always possible - true in the positive rationals, but to apply this law wrongly to the rationals generally. This way of dividing by zero would involve equivocation on the term 'number' and so would be in accord with the One Fallacy Theory (Powers, 1998, p. 656)

However, if another equally (if not more intuitively) plausible explanation of why this fallacy occurs that does not appeal to the role of ambiguity is offered, it seems as though Powers will need to address this explanation and somehow show it to be less plausible than his. Indeed, it seems as though that if this alternate explanation is able to explain why this fallacy is committed by only appealing to the elements of the argument that are present above, (and not by appealing to any other step that the person giving or receiving the argument might be invoking in their head), then that alternate explanation will be more intuitive and plausible.

And it seems as though this can be done. In Powers' explanation, he attempts to posit an extra step the person reviewing the argument might make implicitly in her head which causes her to make the invalid inference from 4 to 5 . This of course occurs because there is an ambiguity involving the concept 'number'. I believe I can explain the invalid step without appealing to any extra ambiguity in the reviewer's head.

What happens when one commits the dividing-by-zero fallacy is that the invalid step seems valid, not because of some ambiguity, but because one does not realize that one is dividing by zero. In this context ' $a-b$ ' represents zero and in canceling ' $a-b$ ' on both sides of the equation the reviewer, consequently, is dividing by zero. He does not forget that one cannot divide by zero; he simply does not realize that ' $a-b$ ' stands, in this context, for zero. Zero is wearing a disguise; the step appears as though it is perfectly valid, though obviously it is not. This explanation solely relies on the appearance of the argument and makes no resort to ambiguity that might be invoked in the reviewer's head. In other words, the notion of number is not itself present in the argument, so why should we claim that it is the source 
of the mistake? If there does not appear to be an ambiguity being relied on by the argument, one should not posit one, unless appeal to the presented elements of the argument does not yield an adequate explanation of the mistake.

Perhaps an analogy can be given. Suppose Bob is a bouncer at a bar, and the owner of the bar tells Bob not to let Slim Shady in because Slim always starts fights when he gets drunk. Bob knows what Slim looks like, he is tall and always clean shaven. So Bob will accordingly restrict Slim from getting into the bar. It just so happens that Bob saw Slim at a costume shop earlier that day buying a fake beard. Bob sees this and doesn't pay much attention to it. Later that evening people start to show up at the bar and Bob, keeping an eye out for Slim, lets them in. Later a drunken bearded guy starts a fight, and Bob comes over to break it up and realizes that the bearded guy is Slim Shady! He has a fake beard on!! He remembers that he saw Slim at the costume shop earlier that day buying a beard, and he knows sometimes people wear fake beards, however it didn't even occur to him that Slim might plan on wearing that fake beard to the bar that night. Joe has made a mistake, but its not a naked mistake, it's a mistake for which he is not obviously culpable. Most likely he is not going to get fired for this mistake. It's not as if Slim walked right up to Bob beardless, and Bob just looked him in the face and let him in. But this type of reasoning seems to apply equally to the dividing-byzero fallacy committed in the above argument. The reviewer of the argument does not make the naked mistake of dividing by zero. That mistake is hidden in a sense from him because zero is masked. In other words, it's not as if the reviewer of the argument divided by ' 0 ' in the argument. Had he done so, then it's clear that it is a naked mistake.

Just because an ambiguity could be found in (or constructed in the explanation of someone's reasoning doesn't itself give us a reason to believe that that is the actual source of the fallacy in general. I believe that this alternate explanation that I have given above is more plausible, because it mainly relies on explanatory elements that come from the information that is provided by the fallacious argument above, and does not require positing extra information that the reviewer of the argument might be implementing. In other words, there does not seem, prima facie, to be an ambiguity at work in the argument as presented. Indeed this alternate explanation shows why the fallacy would be committed by only relying on information that is present in the argument itself. It can explain the same phenomenon, but with few suppositions.

Since Powers' admits that this is a fallacy and since this alternate explanation seems more plausible, it appears as though this might be a counterexample to his theory. He will have to do one of two things: (i) change his mind and try to argue that this is not a fallacy - which does not seem to be viable, since it does appear as though the argument follows the rules of logic or (ii) argue that this alternate explanation is not better and that positing an ambiguity is indeed required to explain why the fallacy is actually committed. 
Now, this alternate explanation does seem to comport with the rational-1/ rational-2 distinction. Since $(a-b)$ does not appear to be zero, one has reason -2 to believe that she can divide by it. This claim relies on what I think is the relatively uncontroversial claim that one can be rational-2, make a mistake, and it not be the case that that mistake be a result of an ambiguity. Rational- 2 people make mistakes in reason other than simply confusing ideas. They make mistakes by not realizing certain important aspects of concepts, and in many cases are justified prima facie in overlooking them. This is a result of having only a fallible grasp of concepts.

It should also be noted that this alternate explanation fits phase II of Powers' metaphilosophy, for an antagonist-philosopher $\mathrm{X}$ can come along and explain why protagonist-philosopher $\mathrm{Y}$ argument is not good, but appears good. Powers says:

My concept of fallacy requires $\mathrm{X}$ and $\mathrm{X}$ 's theory to give an explanation of how a rational person-a rational- 2 person, such as $I$ am assuming $Y$ to bemight be led to think that $Y$ 's argument is a real proof. The concept of fallacy must require that there be such an explanation and that pointing out a fallacy includes pointing out such an explanation, or else that concept of fallacy will do no work in my metaphilosophical theory (Powers 1995, 306).

My explanation does just this. So this alternative explanation does everything that Powers' notion of fallacy asks of it, including on his metaphilosophical view, yet it contains no mention of ambiguity.

The second fallacy that I want to address is the fallacy commonly associated with the Monty Hall Problem. The problem comes from the famous "Let's Make a Deal" show starring Monty Hall. How it goes is that there are three doors: one that has a prize behind it, and two that have nothing at all behind them. Monty, the host, asks you to pick a door at random, explaining to you that after you do so, he will show you what is behind one of the two remaining doors. After he does this, he will ask you if you want to switch from the door that you originally chose to the remaining closed door. The question is: do your chances of picking the door with the prize behind it get better, worse or stay the same if you switch doors? The correct, yet counterintuitive solution, is that your chances of winning the prize in fact double from $1 / 3$ to $2 / 3$ if you switch to the remaining door. The reasoning for one solution to this problem is subtle, and involves notions of probability theory. The reasoning involved in another solution does not require that one understand such theorems to see why her intuitive solution is incorrect.

Suppose that the game has begun and that the prize is behind door A. This is, of course, unbeknownst to you, the contestant. So there are two wrong doors: B and C. Let's suppose that you take the strategy of never switching doors. Your chances of picking the correct door are $1 / 3$ overall. The chance that you will lose, however, is $2 / 3$. But now what if you always take the strategy of switching doors? If you pick door A, then you lose. If you pick door B, you win. And if you pick door C, you win. So your chances of winning, if you always switch, are 2/ 
3. Now, our picking of door A as the winning door was, of course, random and makes no difference to the outcome of the game. In other words, one could have just as well called the doors 'winning door', 'losing door 1' and 'losing door 2'. Therefore, in general, if you always switch, your chances of winning will be $2 / 3$ and if you never switch, your chances of winning will always be 1/3.

Now, the intuitive, yet incorrect, answer seems to be that your chances of winning the prize stay the same whether you stay or switch-it's 50-50! After all, there are two doors and the prize is behind one of the doors, many figure it's just like flipping a coin! This seems to be the rationale that one goes through when trying to figure out the answer to this problem. One thinks: "Since there are three doors and the prize is behind only one door, there is a $1 / 3$ chance of picking the door with the prize behind it." (Then Monty eliminates a door.) Now one reasons: "Since there are two doors and the prize is only behind one door, there is a $1 / 2$ chance of picking the door with the prize behind it. Therefore, the odds of the prize being behind each remaining door are exactly the same; 50-50." But here again, there does not seem to be any ambiguity at work. It's simply not enough to try to find an explanation involving ambiguity. It doesn't seem as though Powers, when confronted with a potential counterexample, should try to develop an explanation involving ambiguity, when a fallacy does not seem, prima facie, to contain one. This mistake seems to occur because of a lack of understanding how probabilities work in conjunction with the thought that the principle that one is invoking is obviously true. Here the overlooking is completely reasonable and rational. Many do not understand what counts as relevant evidence and how that figures into the calculations of the probabilities. Furthermore, people seem to treat what they see as relevant cases relevantly. In other words, when they are presented with the two doors and are asked whether or not they would like to switch, the only relevant information they see is that there are two doors and one prize; therefore 50-50. Note that it is not the case that they are making an argument by analogy; it's that they think that the rules of chance are such that their reasoning is obviously correct. They don't understand, how certain pieces of evidence come into play in calculating probabilities. But it's not a naked mistake that they make. They are justified in making the mistake, because they lack evidence to think that they are wrong, and they believe, falsely, that they are obviously correct. They are not epistemically blameworthy in their overlooking of the relevant information. Again, the mistake is covered, but apparently not by any ambiguity.

It's hard to see how there could be an ambiguity in this Monty Hall fallacy. I believe that I have given the actual explanation of why the fallacy is committed; many justifiably believe that they are using correct inference rules, when they are not. Furthermore, the inference they make has an incredibly strong appearance of validity in Powers' sense.

The last fallacy that I want to consider is the fallacy committed by failing the Wason Selection Test, created by Wason (1966). Accordingly, I will be referring to it as the Wason Fallacy. There are four cards lying on a table in front of you. 
You know for certain that all the cards have one letter marked on one side, and one number marked on the other. Marked on their upper sides might be the following:

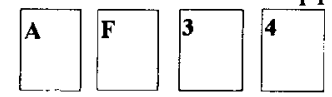

There is a statement: "If a card has a vowel on one side, then it must have an even number on the other side." How many cards need one turn over, and which cards, to establish whether the statement is true or false?

Most people ( $75 \%-80 \%$ of test takers) including those trained in formal logic answer incorrectly. Many people select the card with ' $A$ ' on its upper side as the only card that you need to flip over. To see if a conditional is false one needs to see if there is a case when the antecedent is true and the consequent is false. But in this case, people do not realize that flipping the card with the ' $A$ ' on it is only half of the work, they must also flip over the card with the ' 3 ' on it. Remember the statement is "If a card has a vowel on one side, then it must have an even number on the other side" and can be represented as $\mathrm{P} \supset \mathrm{Q}$, but the logically equivalent statement $\sim Q \supset \sim P$ must also be tested and to do that one must flip the ' 3 ' card. This fallacy seems to occur, once again, because people overlook certain important information. They realize they need to flip the card that satisfies the antecedent, but they do not realize that they must also flip over the card with the denial of the consequent. There does not seem to be an ambiguity involved in making this mistake, and indeed it seems as though rational-2 people in fact commit this fallacy. This seems to be a further problem for Powers' theory, for the incorrect solution does seem as though it follows the rules of logic (thus a legitimate fallacy on Powers' terms) and yet there does not appear to be, prima facie, any ambiguity at work. Furthermore, this overlooking seems non-culpable because the standard way of checking the truth-value of a conditional is not being employed, thus when one approaches this problem they are tricked into believing that their selection of ' $A$ ' is the only card that needs to be flipped. The reason, in this case, that one is non-culpable for making this mistake seems to be in part because of the strong appearance of validity in answering the question "half" correctly.

All these fallacies, I argue, are the result of not realizing, or overlooking, information that is important to drawing the appropriate conclusion. One can make mistakes, while at the same time possessing rationality-2, not only because one does not see an ambiguity, but also because one justifiably overlooks or does not recognize certain important aspects of some concept. This is part of what it is to have an incomplete grasp of a concept.

Powers might reply that people overlook all kinds of things, and by including overlooking relevant information as a fallacy almost anything will count as a fallacy. Since I agree that not just any overlooking should be considered a fallacy, I give two criteria for what kind of overlooking should count as a fallacy: an overlooking results in the committing of a fallacy if and only if (i) the overlooking is the result of the appearance of validity in the sense that the conclusion appears to follow from the premises by the laws of logic, and (ii) the overlooking is epistemically non-culpable. 


\section{Matthew Zuckero}

To wrap up, my case is as follows: I have given three fallacious arguments (with explanations that do not involve ambiguity) that I believe to be counterexamples to Powers' One-Fallacy Theory. I concluded that these are counterexamples to his theory for the following reasons:

(i) If there is, prima facie, no ambiguity present in a fallacious argument, then one should be posited in explaining the fallaciousness of that argument if and only if it is necessary for explaining why that argument is fallacious.

(ii) There is, prima facie, no ambiguity present in the fallacious arguments I have listed in this paper as potential counterexamples.

(iii) Therefore, an ambiguity should be posited in the explanation of the fallacious arguments above if and only if it is necessary for explaining why those arguments are fallacious.

(iv) If there is, ceteris paribus, a simpler explanation of a fallacy that does not involve ambiguity, then it is unnecessary to posit an ambiguity in explaining that fallacy.

(v) There is, ceteris paribus, a simpler explanation of the fallacious arguments above that does not involve ambiguity.

(vi) Therefore, it is unnecessary to posit an ambiguity in explaining the fallacious arguments above.

(vii) Therefore, an ambiguity should not be posited in explaining why the above arguments are fallacious.

(viii) If there is a fallacious argument in which an ambiguity should not be posited in explaining why it is fallacious, then the One-Fallacy Theory is false.

(ix) Therefore, the One-Fallacy Theory is false.

\section{References}

Powers, Lawrence H. (1999). "Dividing by Zero and Other Mathematical Fallacies" in Proceedings of the International Society for the Study of Argumentation, van Eemeren et al., eds. Amsterdam: SicSat, pp. 655-57.

Powers, Lawrence H. (1995). "The One Fallacy Theory," Informal Logic, 17.2, 303-314.

Wason, P. C. (1966). "Reasoning," in New Horizons in Psychology, B. M. Foss, ed. Hammondsworth: Penguin. pp. 135-51. 